\title{
RESISTIVITY MEASUREMENT TECHNIQUES FOR COMPOSITE
}

\section{MATERIALS}

\author{
Florita D'Sa ${ }^{1}$, Shubha $V^{2}$, Anil Kumar $A^{3}$ \\ ${ }^{1}$ Scientist, Airport and Thermophysical Instrumentation Group, National Aerospace Laboratories, Bangalore, India \\ ${ }^{2}$ Chief Scientist, Airport and Thermophysical Instrumentation Group, National Aerospace Laboratories, Bangalore, \\ India \\ ${ }^{3}$ Senior Scientist, Advanced Composites Division, National Aerospace Laboratories, Bangalore, India
}

\begin{abstract}
In this article, the theoretical concepts and the experimental techniques for measurements of the volume and surface resistivity of carbon fibre reinforced polymer (CFRP) composite materials have been explained. The purpose of these measurements is to investigate the potential of composite materials as lightning arrestors for an aircraft. In particular, the AC resistivity method and the Delta mode of measurement have been described in detail. A few measurements were carried out using an indigenously designed and fabricated collinear four-probe resistivity measurement tool, for which factors dependent on the dimensions of the samples have to be incorporated, as per the ASTM F390-11 standards. The validity of the two-probe and four-probe methods is also analyzed, along with techniques to minimize errors in measurements.

Conductivity measurements on uni-directional laminates have been made in the longitudinal (fibre) direction as well as perpendicular to it, indicating a marked difference in the relative magnitudes. When investigating the potential of materials as lightning arrestors, a very high surface conductivity is desired so that lightning currents (even of the order of kA) pass along the exterior of the aircraft, without penetrating the underlying structure. The effects of the addition of copper nanotubes (CNT) and copper mesh have also been studied. The instruments involved in these experiments have been automated based on the IEEE 488.2 protocols. The software to program them was developed using Labview. Measurements have also been performed on standard samples to test the accuracy of the data. Currents ranging from 0.1 to $100 \mathrm{~mA}$ were passed, to deduce the currentdependency of the surface resistivity of these materials.
\end{abstract}

Keywords: CNT, Labview, Delta, GPIB

\section{INTRODUCTION}

Measurements of surface resistivity are required when a conductive coating is present on an insulator, in which case the magnitude of the resistivity of the surface is distinctly different from that of the bulk of the material. The volume resistivity of a material is also referred to as its bulk resistivity since it is determined by the entire volume of the material.

The two-probe method for the measurement of resistivity is adequate if the resistance of the contacts (or leads) is much lower than the specimen's resistance, but if it is commensurate with that of the specimen, this method would lead to errors in measurements. Errors may also arise due to thermoelectric voltages, when conductors made of dissimilar metals are joined together. In order to avoid these problems, the four-probe method is employed, using either alternating current or a rapidly reversed direct current. To ensure that the current density in the sample is uniform, the current and the voltage contacts should not be very closely spaced [1]. If connecting surfaces are not kept clean and free of oxides, a copper-to-copper oxide junction could result in a thermoemf as high as $1 \mathrm{mV} /{ }^{\circ} \mathrm{C}$.

Methodology: The three different techniques employed for the measurements are explained in the following section.
Four-probe collinear measurements: A few measurements were carried out using an indigenously designed and fabricated measurement tool. This tool ensures that the four collinear probes touch the sample only at their tips and the points of measurement are in the middle of the sample.

Delta measurements: For such measurements, the applied current is repeatedly reversed to nullify noise due to thermoelectric effects. With sophisticated data acquisition instruments, a high speed of reversal is possible and moving averages are calculated using three values at a time, instead of only two, to enhance the quality of the data, as illustrated in Figure 1 [2].

AC Measurements (lock-in technique): This is another technique employed for the measurement of resistivity, whereby a signal from the sample is locked to a reference signal, derived from the same current source. The operation of a lock-in amplifier (also referred to as a phase-sensitive detector) relies on the orthogonality of sinusoidal functions. Accurate measurements can be made even when the signal of interest is obscured by much larger noise sources. Measurements are usually made at a frequency of $400 \mathrm{~Hz}$, to avoid line-frequency interference. 


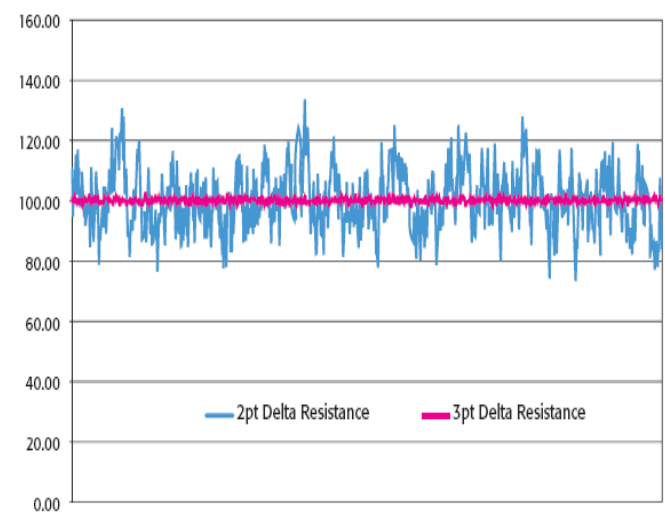

Fig 1: A comparison of the 2-point and 3-point Delta techniques

\section{EXPERIMENTATION}

A programmable current source is used for currents up to $100 \mathrm{~mA}$, with a voltage compliance of $105 \mathrm{~V}$. The current source works with either a GPIB or an RS232 interface. For experiments at higher currents, a $60 \mathrm{~V}, 5 \mathrm{~A}$ power supply is employed, in conjunction with a digital multimeter. The instruments are controlled by a computer using Standard Commands for Programmable Instruments (SCPI), an extension of the IEEE-488.2 protocol. Software has been written in Labview (by the first author) to automate deltamode resistivity measurements, with features to improve the quality of the data such as altering the number of power line cycles. Setting the measurement speed of the A/D converter involves a compromise between speed and noise. Line cycle synchronization can also be enabled to enhance the rejection of common-mode noise.

To verify the accuracy of the system, experiments have been performed on bars of pure copper, whereby it was noticed that the resistance varied by just 2 to $3 \mu \Omega$, over a wide range of currents.

\section{RESULTS AND CONCLUSIONS}

(1) Resistance measurements carried out on different composite materials by employing the Delta and the AC methods are listed in Table 1, clearly indicating that the values are almost identical.

Table 1: Comparing the resistances measured by two different techniques

\begin{tabular}{|l|l|}
\hline $\begin{array}{l}\text { Delta Method) } \\
\text { Resistance }(\mathrm{m} \Omega)\end{array}$ & $\begin{array}{l}(\mathrm{AC} \text { technique }) \\
\text { Resistance }(\mathrm{m} \Omega)\end{array}$ \\
\hline 392.695 & 391.93 \\
\hline 128.235 & 128.33 \\
\hline 111.645 & 111.295 \\
\hline
\end{tabular}

(2) The measurements listed in Table 2 were made on a 300x300x2 mm unidirectional (UDCFRP) laminate with electrodes placed at different positions using silver paste, as depicted in Figure 2.

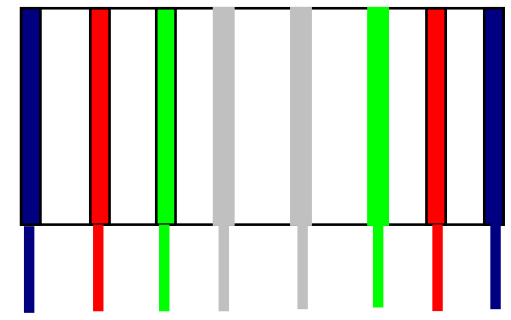

Fig 2: Leads positioned on a UDCFRP laminate for resistivity measurements [3]

Table 2: Volume resistivity measurements on a UDCFRP panel

\begin{tabular}{|l|l|}
\hline $\begin{array}{l}\text { Resistance/length } \\
\text { ratio }(\mathrm{m} \Omega / \mathrm{cm})\end{array}$ & $\begin{array}{l}\text { Volume } \\
\text { resistivity } \\
(\mathrm{m} \Omega . \mathrm{cm})\end{array}$ \\
\hline 4.352 & 24.3712 \\
\hline 3.984 & 22.3104 \\
\hline 4.065 & 22.764 \\
\hline 3.89 & 21.784 \\
\hline
\end{tabular}

The ratio of the resistances measured in different positions is approximately equal to the corresponding ratio of the distances between the voltage leads, thus proving that the longitudinal resistance in the direction of the fibre varies linearly with the length of the specimen [4].

(3) Surface Resistivity measurements: Measurements of surface resistivity are required when either a conductive coating is applied on an insulator or a non-conductive layer is present on the surface of a conductor. Since the resistance of only the surface is under investigation, the volume of the sample does not feature in any calculations. Surface resistivity is determined by the ratio of the voltage drop per unit length to the surface current per unit width.

It has been noticed that the surface resistivity of materials that aren't very good conductors is dependent on the applied voltage. In the case of such materials, the measured surface resistance is relatively high when low currents are passed, but falls considerably with the passage of higher currents. Figure 3 depicts the variation of the surface resistivity as a function of current, for an epoxy primer sample with $8 \%$ CNT, while Figure 4 is the plot of the surface resistivity for a similar epoxy primer sample, but with an additional coating of 4 layers. 


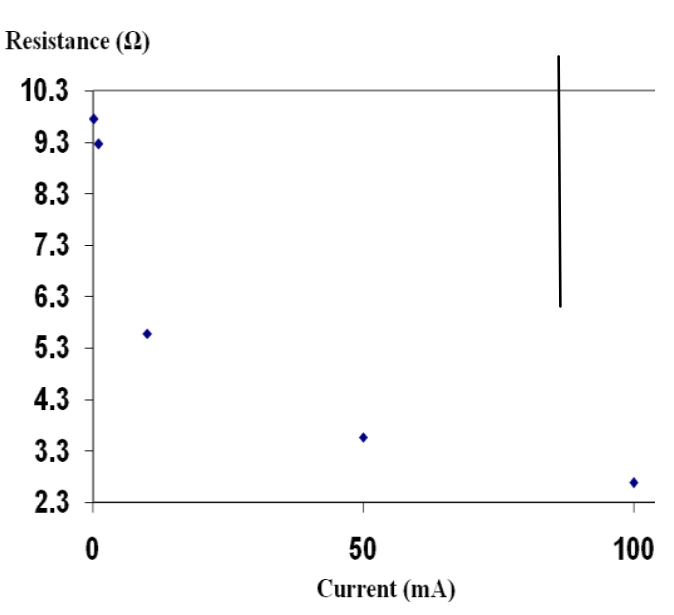

Fig 3: The surface resistance of a composite material falls steeply with increasing current

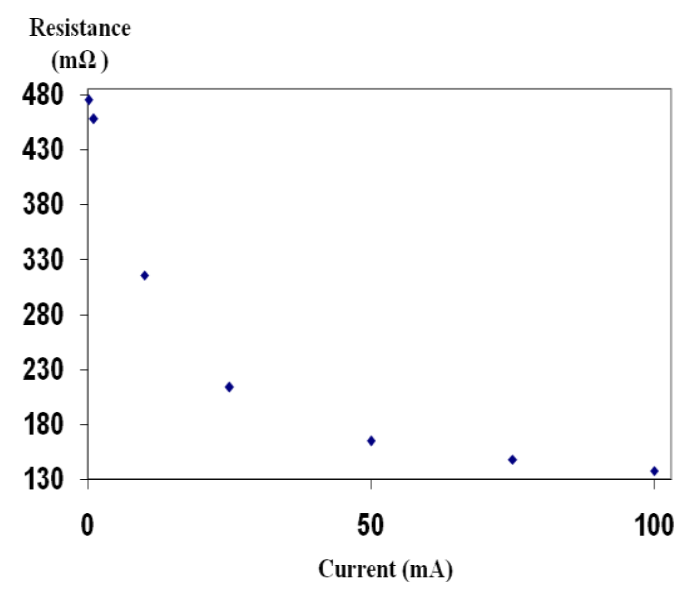

Fig 4: Higher currents lower the surface resistance of a composite sample

At higher potentials, the layers of the material below the surface begin to conduct current. This effect creates resistances in parallel with the surface resistance, thus lowering its value.

(4) The effect of CNT was investigated using nonconducting samples, as indicated in Table 3.

Table 3: Effects of different proportions of CNT on nonconducting samples

\begin{tabular}{|l|l|l|}
\hline $\begin{array}{l}\text { \% (weight) } \\
\text { of CNT in a } \\
\text { non- } \\
\text { conducting } \\
\text { sample }\end{array}$ & $\begin{array}{l}\text { Resistance } \\
\text { of the } \\
\text { sample }\end{array}$ & Remarks \\
\hline 0 & $7.843 \mathrm{M} \Omega$ & \\
\hline 0.1 & $7.539 \mathrm{M} \Omega$ & $\begin{array}{l}\text { The ratio of the } \\
\text { decrease in } \\
\text { resistance to the } \\
\text { previous value of } \\
\text { resistance is } \\
93.2 \%\end{array}$ \\
\hline 0.5 & $0.509 \mathrm{M} \Omega$ \\
\hline
\end{tabular}

\begin{tabular}{|l|l|l|}
\hline 1 & $\begin{array}{l}43.261 \\
\mathrm{~K} \Omega\end{array}$ & $\begin{array}{l}\text { The ratio of the } \\
\text { decrease in } \\
\text { resistance to the } \\
\text { previous value of } \\
\text { resistance is } \\
91.5 \%\end{array}$ \\
\hline
\end{tabular}

(5) Measurements were also carried out on uni-directional CFRP samples with identical dimensions and compositions, but with currents passed parallel to as well as perpendicular to the direction of the fibre. In the case of the former, the resistance was of the order of $\mathrm{m} \Omega$, while in the case of the latter, it was of the order of ohms. These results prove that the longitudinal conductivity of these composites is considerably higher than the transverse conductivity [4].

(6) Table 4 depicts the reduction in the volume resistance of a CFRP laminate meant for lightning tests, due to the inclusion of copper mesh.

Table 4: The reduction in the volume resistance after the addition of copper mesh

\begin{tabular}{|l|l|}
\hline $\begin{array}{l}\text { Resistance in m } \\
\text { without copper mesh } \\
\text { (currents ranging } \\
\text { from 1-4 Amps) }\end{array}$ & $\begin{array}{l}\text { Resistance in } \mathrm{m} \Omega \\
\text { with copper mesh } \\
\text { (currents ranging } \\
\text { from 1 - 4 Amps) }\end{array}$ \\
\hline 17.55 & 4.759 \\
\hline 12.24475 & 2.885 \\
\hline 6.326 & 1.6465 \\
\hline 2.56825 & 0.4945 \\
\hline
\end{tabular}

(7) Collinear measurements:

(1) The accuracy of this method has been established by performing measurements on strips of aluminium foil.

(2) Four-probe collinear resistivity measurements were carried out on a composite sample infused with copper mesh and factors dependent on the dimensions of the sample were calculated, as per the ASTM F390-11 standards. The addition of the copper mesh lowered the volume resistivity of the composite material to $873 \mu \Omega \mathrm{m}$.

(3) Measurements were also made on samples consisting of different insulating substrates, each with a 10 micron coating of nickel. The addition of nickel reduced the volume resistivity of these materials to the range $2627-4237 \mu \Omega \mathrm{m}$.

\section{ACKNOWLEDGEMENTS}

The authors thank Mr Jagannath Rao for his help in the fabrication of the four-probe collinear measurement setup.

\section{REFERENCES}

[1]. Shen, L., J. Li, B. M. Liaw, F. Delale and J. H. Chung, "Model and analysis of the electrical resistance measurement of carbon fiber polymer-matrix composites", Composites Science and Technology, 67, 2007, pp. 25132520 .

[2]. Daire, A. and W. Goeke, "New instruments can lock out lock-ins”, Keithley Instruments Application Notes, 2006. 
[3]. D'Sa, F. and V. Shubha, "Volume and Surface Resistivity Techniques for Composite Materials", N.A.L. Project Document, January 2012.

[4]. Abry, J.C., S. Bochard, A. Chateauminois, M. Salvia and G. Giraud, "In-situ detection of damage in CFRP laminates by electrical resistance measurements", Composites Science and Technology, 59, 1999.

\section{BIOGRAPHIES}

Florita D'Sa has an M.Sc. in Physics and is a scientist in the Airport and Thermophysical Instrumentation Group at NAL. Her work involves data-acquisition as well as a range of thermo-physical measurements. She began her career as a Research Intern at Raman Research Institute in Bangalore. Her email id is fdsa@nal.res.in

V. Shubha has a Ph.D. in the field of high-pressure instrumentation and is the Head of the Airport and Thermophysical Instrumentation Group, NAL. She has won several awards for her work in the field of visibility measurements for airports as well as thermophysical instrumentation. Her email id is vshubha@nal.res.in

A. Anil Kumar has a Ph.D. in the field of polymer sciences and is currently a senior scientist in the Advanced Composites Division at NAL. His email id is aanil@nal.res.in 